\title{
Worst Case and Expected Peak-to-Average Power Ratio for Orthonormal Systems
}

\author{
Brendan Farrell and Holger Boche \\ Lehrstuhl für Theoretische Informationstechnik \\ Technische Universität München \\ Arcisstr. 21, 80333 München, Germany \\ Email: farrell@tum.de,boche@tum.de
}

\begin{abstract}
We present several results that show, according to several criteria, that the large peaks that occur in OFDM are common to all orthonormal systems that are uniformly bounded. In particular, worst case peak-to-average values are always at least the square-root of the number of signals, and the expected peak value of a signal always increases logarithmically with the number of signals. The same growth also occurs for single-user signals with the appropriate normalization. Further, we investigate peak-to-average power properties of the prolate spheroidal wave functions and the Walsh functions, which are used in DS-CDMA.
\end{abstract}

\section{INTRODUCTION}

Large signal peak values are one of the main drawbacks of OFDM systems and, thus, their study is currently an area of much research. In this paper we show in a number of statements that the behavior seen in the OFDM system is common to all bounded orthonormal systems. This is true of typical single-user as well as multi-carrier systems. In fact, OFDM offers ideal performance by several measures, in that its behavior achieves the best possible. This is true for the worst-case deterministic peak as well as the expected value of the peaks. We show that analogous behavior occurs for the set of prolate spheroidal wave functions and for the Walsh functions, which are the signals used in DS-CDMA. These observations are timely since it is expected that $5 \mathrm{G}$ wireless systems will move from strict OFDM signals to other waveforms.

We begin Section II by showing that the peak-values of single-user signals can grow logarithmically with the signal length. We then give several propositions that demonstrate that all orthonormal systems can lead to peak values of at least the same order as OFDM signals. We give several variations of this result. In the OFDM case, it is well-known that expected signal peaks grow logarithmically in the number of signals. We show that a powerful result from probability in Banach spaces yields the same behavior for all uniformly bounded orthonormal systems. This shows that there does not exist another orthonormal systems that immediately has a better peak value behavior than the OFDM system. In

B. Farrell was supported by the German Science Foundation (DFG) under Project BO 1734/18-1, and H. Boche was supported by a start-up fund of the Technische Universität München. doing so, we hope to make a significant mathematical result with direct application to multi-carrier systems familiar to the communications community. In Section III we address peak value properties of the prolate spheroidal wave functions. There are two motivations for doing this. First, they are defined for long time intervals, and so it is valuable to determine if they have enough overlap among signals to lead to a high peak value. Secondly, these signals were used by Pollak, Slepian and Landau to prove Shannon's fundamental theorems of information theory. Proving that they have a strong peak growth then means that the approach taken by Pollak, Slepian and Landau would be insufficient if a peak value constraint were imposed. In Section IV we address the signals used in DS-CDMA, namely the Walsh functions. While the results of Section II apply to DS-CDMA, we show explicitly how peak values of the square-root of the number of signals can occur there. We use 'Theorem' to denote results cited from the mathematics literature and 'Lemma' or 'Proposition' for our results.

\section{Peak VAlues for Linear Combinations of ORTHONORMAL SIGNALS}

\section{A. Worst Case Behavior}

Most of this paper deals with multi-user communication systems, and the central statement is that peak values grow logarithmically with respect to the number of users. Before getting into the multi-user setting, we observe that the same peak growth also occurs in the single-user setting.

Proposition 2.1: Assume that the single-user transmission signal is a linear combination of integer shifts of $\frac{\sin \pi(t)}{\pi t}$ with coefficients drawn from the alphabet $\{ \pm 1\}$. Then

$$
\sup _{a_{n}= \pm 1, n=0, \ldots, N-1} \sup _{t \in \mathbb{R}}\left|\sum_{n=0}^{N-1} a_{n} \frac{\sin \pi(t-n)}{\pi(t-n)}\right| \geq \frac{1}{\pi} \log (N)
$$

Proof: Let $t_{0}$ be the point in $[0, N]$ where $\sum_{n=0}^{N-1}\left|\frac{\sin \pi(t-n)}{\pi(t-n)}\right|$ is maximized, and set $a_{n}=$ $\operatorname{sign}\left[\frac{\sin \pi\left(t_{0}-n\right)}{\pi\left(t_{0}-n\right)}\right]$. Then, by using the lower bound given by setting $t_{0}=1 / 2$,

$$
\sup _{a_{n}= \pm 1, n=0, \ldots, N-1} \sup _{t \in \mathbb{R}}\left|\sum_{n=0}^{N-1} a_{n} \frac{\sin \pi(t-n)}{\pi(t-n)}\right|
$$




$$
\begin{aligned}
& \geq \sum_{n=0}^{N-1}\left|\frac{\sin \pi\left(t_{0}-n\right)}{\pi\left(t_{0}-n\right)}\right| \\
& \geq \sum_{n=0}^{N-1}\left|\frac{\sin \pi\left(\frac{1}{2}-n\right)}{\pi\left(\frac{1}{2}-n\right)}\right| \\
& =\frac{1}{\pi} \sum_{n=0}^{N-1} \frac{1}{\left|n-\frac{1}{2}\right|} \\
& \geq \frac{1}{\pi} \sum_{n=1}^{N} \frac{1}{n} \\
& \geq \frac{1}{\pi} \log (N) .
\end{aligned}
$$

Since the signal in the above proposition is very sensitive to time jitters, one may consider the more robust signal

$$
s(t)=t \sum_{n=1}^{N} \frac{a_{n}}{n} \frac{\sin \pi(t-n)}{\pi(t-n)} .
$$

However, by essentially the same calculation as in the proof above, by taking $t_{0}=N-1 / 2$, one easily sees that this signal also can have peaks that grow logarithmically with $N$.

We note that there is a normalization implicit in the previous proposition of $\sqrt{N}$, that is the $L^{2}([0,1])$-norm of the signal above is $\sqrt{N}$. In the single-user setting, normalizing with respect to the time-duration of the signal is appropriate, and this is done in the formulation above. In the multi-user setting, where $N$ coefficients are transmitted not by $N$ time steps but by placing $N$ functions in one time interval, the correct normalization is the $l^{2}$-norm of the coefficients. Thus, the growth in these two cases has different causes. First we formalize the peak-to-average power ratio for multi-carrier systems.

Definition 2.2: For a set of functions $\left\{f_{n}\right\}_{n=1}^{N} \subset L^{2}([0,1])$ we define the general peak-to-average-power ratio

$$
\operatorname{gapr}\left(\left\{f_{n}\right\}_{n=1}^{N}\right)=\sup _{a \in l_{N}^{2}} \frac{\left\|\sum_{n=1}^{N} a_{n} f_{n}\right\|_{L^{\infty}([0,1])}}{\|a\|_{l_{N}^{2}}}
$$

The following lemma gives the worst-case behavior.

Lemma 2.3: (See Theorem 6 in [1]) For any $\left\{f_{n}\right\}_{n=1}^{N} \subset$ $L^{2}([0,1])$ satisfying $\left\|f_{n}\right\|_{L^{2}([0,1])}=1$ for all $n$,

$$
\sqrt{N} \leq \operatorname{gapr}\left(\left\{f_{n}\right\}_{n=1}^{N}\right)=\operatorname{ess.sup} \operatorname{su}_{t \in[0,1]}\left(\sum_{n=1}^{N}\left|f_{n}(t)\right|^{2}\right)^{1 / 2} .
$$

It is helpful to compare Lemma 2.3 with what is known for both OFDM and DS-CDMA signals. In both of those cases, the upper bound on gapr is $\sqrt{N}$, so that in these two cases gapr $=\sqrt{N}$. Lemma 2.3 then shows that the behavior of the OFDM and DS-CDMA signal sets is in fact optimal in this aspect and, in fact, can be seen as a lower bound for all other orthonormal systems. This can be seen as a correction to the notion that high peak values is a problem particular to the OFDM system.
The proof of the previous lemma makes use of specifically selected coefficients. In general, though, coefficients are drawn from a finite alphabet, and so one must determine if the same behavior can occur with this restriction on the coefficients. The following proposition shows that this is indeed the case, with a scaling due to the maximum magnitude of the signals.

Proposition 2.4: Assume the transmission signals $\left\{f_{n}\right\}_{n=1}^{N}$ are $\|\cdot\|_{L^{2}([0,1])}$-normalized and are also uniformly bounded by $M$, and that at least one signal achieves this bound. For all $N$ there exists a sequence $\left\{a_{n}\right\}_{n=1}^{N} \in l_{N}^{2}$ satisfying $\|a\|_{l_{N}^{2}}=\sqrt{N}$ such that

$$
\sup _{t \in[0,1]}\left|\frac{1}{\sqrt{N}} \sum_{n=1}^{N} a_{n} f_{n}(t)\right| \geq \max \left(M, \frac{\sqrt{N}}{2 M}\right) .
$$

The bound $\sqrt{N} / 2 M$ can be established while requiring $a_{i} \in$ $\{ \pm 1\}$ for $i=1, \ldots, N$.

We point out the different assumptions on the coefficient alphabets in Propositions 2.1 and 2.4. If we only assume that $\|a\|_{l_{N}^{2}}=\sqrt{N}$ in Proposition 2.1, then we would trivially have peaks of size $\sqrt{N}$. Thus, there the proposition points out that peak growth still occurs with coefficients taking the values $\{ \pm 1\}$. In Proposition 2.4, if we also required the coefficients be in $\{ \pm 1\}$, then we would obtain the lower bound $\frac{\sqrt{N}}{2 M}$, as can be seen from the proof below.

Proof: Write each $f_{n}$ as $f_{n}(t)=u_{n}(t)+i v_{n}(t)$, where $u_{n}$ and $v_{n}$ are real. Then

$$
\begin{aligned}
N & =\int_{0}^{1} \sum_{n=1}^{N}\left|f_{n}(t)\right|^{2} d t \\
& =\int_{0}^{1} \sum_{n=1}^{N}\left|u_{n}(t)\right|^{2}+\left|v_{n}(t)\right|^{2} d t
\end{aligned}
$$

which means either $\int_{0}^{1} \sum_{n=1}^{N}\left|u_{n}(t)\right|^{2}>N / 2$ or $\int_{0}^{1} \sum_{n=1}^{N}\left|v_{n}(t)\right|^{2}>N / 2$. Without loss of generality we assume that the former is the case. Then

$$
\begin{aligned}
& \sup _{a \in l_{N}^{2},\|a\|_{l_{N}^{2}}=\sqrt{N}} \sup _{t \in[0,1]}\left|\sum_{n=1}^{N} a_{n} f_{n}(t)\right| \\
\geq & \sup _{a_{n}= \pm 1, n=1, \ldots, N} \sup _{t \in[0,1]}\left|\sum_{n=1}^{N} a_{n} u_{n}(t)\right| \\
\geq & \sup _{t \in[0,1]}\left|\sum_{n=1}^{N} \operatorname{sign}\left(u_{n}(t)\right) u_{n}(t)\right| \\
= & \sup _{t \in[0,1]} \sum_{n=1}^{N} \operatorname{sign}\left(u_{n}(t)\right) u_{n}(t) \\
\geq & \sup _{t \in[0,1]} \frac{1}{M} \sum_{n=1}^{N} u_{n}(t)^{2} \\
\geq & \frac{1}{M} \int_{0}^{1} \sum_{n=1}^{N} u_{n}(t)^{2} d t \\
\geq & \frac{N}{2 M} .
\end{aligned}
$$


Dividing by $\sqrt{N}$ shows that a peak of size $\frac{\sqrt{N}}{2 M}$ can be obtained. Of course, we may also construct a signal that achieves the bound $M$ by making the coefficient of the appropriate transmission signal $\sqrt{N}$ and all the others zero.

\section{B. Expected Behavior}

It is well-known in the OFDM case that if one has coefficients drawn from a finite alphabet with expected value 0 , then the discrete Fourier transform applied to these coefficients results in random variables that approach a Gaussian random variable by the central limit theorem. The expected value of the maximum of $N$ independent Gaussian random variables grows with order $\log (N)$, and in fact most coefficient vectors result in a maximum on the order of $\log (N)$. Thus, for the Fourier basis, most coefficient vectors result in peaks growing like $\log (N)$. The following theorem, due to Kashin and Tzafriri, shows that the same behavior occurs for any orthonormal system that is uniformly bounded. This theorem is quite fundamental and has a sophisticated 12 page proof.

Theorem 2.5: (Main theorem in [2]) Let $f_{n}:[0,1] \rightarrow \mathbb{C}$, $n=1,2, \ldots$ be orthonormal functions in $L^{2}([0,1])$ satisfying $\left\|f_{n}\right\|_{L^{3}([0,1])} \leq A$ for every $n$, and assume $X_{1}, X_{2}, \ldots$ are independent random variables such that

$$
\mathbb{E} X_{n}=0, \quad \mathbb{E} X_{n}^{2}=1, \quad\left(\mathbb{E}\left|X_{n}\right|^{3}\right)^{1 / 3} \leq A
$$

for all $n$. Then there exists a constant $K>0$ depending only on $A$ such that

$$
\mathbb{E} \sup _{t \in[0,1]}\left|\frac{1}{\sqrt{N}} \sum_{n=1}^{N} X_{n} f_{n}(t)\right| \geq K \sqrt{\log N} .
$$

In principle, the expectation above could increase like $\sqrt{\log (N)}$ while for most coefficient sequences it remains bounded. The following corollary, however, shows that most coefficient sequences lead to peaks of order $\sqrt{\log (N)}$. The corollary makes use of a standard concentration of measure argument.

Corollary 2.6: Let $f_{n}$ satisfy the conditions of Theorem 2.5 as well as $\left\|f_{n}\right\|_{L^{\infty}([0,1])} \leq M$ for all $n=1,2, \ldots$. Let $X_{n}=$ $\pm P$ each with probability $1 / 2$ for $n=1,2, \ldots$, . Then there exists a constant $K$ depending only on $M$ and $P$ such that

$\mathbb{P}\left(\sup _{t \in[0,1]}\left|\frac{1}{\sqrt{N}} \sum_{n=1}^{N} X_{n} f_{n}(t)\right|<K \sqrt{\log N}-r\right) \leq e^{-r^{2} / 4 P^{2} M^{2}}$.

Proof: For $X, Y \in\{P,-P\}^{N}$ we define the distance $d(X, Y)=\sharp\left\{n \in\{1, \ldots, N\}: X_{n} \neq Y_{n}\right\}$. Set

$$
F(X)=\sup _{t \in[0,1]}\left|\frac{1}{\sqrt{N}} \sum_{n=1}^{N} X_{n} f_{n}(t)\right| .
$$

Then

$$
\frac{|F(X)-F(Y)|}{d(X, Y)} \leq \frac{\frac{2 P M}{\sqrt{N}} d(X, Y)}{d(X, Y)}=\frac{2 P M}{\sqrt{N}} .
$$

We then apply Corollary 1.17 in [4]. That is, we have that $F$ is $\frac{2 P M}{\sqrt{N}}$-Lipschitz, the diameter of each space $\left(X_{N}, d_{N}\right)$ is 1 , and the sum of the diameters squared is $D^{2}$. Using $-F$ where Ledoux writes $F$, and using Theorem 2.5, we have

$$
\begin{aligned}
& e^{-r^{2} / 4 P^{2} M^{2}} \\
& \geq \mathbb{P}\left(\sup _{t \in[0,1]}\left|\frac{1}{\sqrt{N}} \sum_{n=1}^{N} X_{n} f_{n}(t)\right|\right. \\
& \left.\geq \mathbb{E} \sup _{t \in[0,1]}\left|\frac{1}{\sqrt{N}} \sum_{n=1}^{N} X_{n} f_{n}(t)\right|-r\right) \\
& \geq \mathbb{P}\left(\sup _{t \in[0,1]}\left|\frac{1}{\sqrt{N}} \sum_{n=1}^{N} X_{n} f_{n}(t)\right|<K \sqrt{\log N}-r\right) .
\end{aligned}
$$

The previous results have all dealt with signals defined on $[0,1]$, but of course similar results hold for any sampling period. A sufficient condition for growth of gapr of order $\sqrt{N}$ is that the functions $\left\{f_{n}\right\}_{n=1}^{N}$ have sufficient overlap. This is shown by the next lemma. While the lemma is very simple, the proposition following it shows that the simple observation is quite meaningful; the property in the frequency domain is essential for the prolate spheroidal wave functions later.

Lemma 2.7: If there exists a constant $C_{1}$ and an interval $\left[0, T_{s}\right]$ such that

$$
\int_{0}^{T_{s}}\left|f_{n}(t)\right|^{2} d t \geq C_{1}
$$

or

$$
\int_{0}^{T_{s}}\left|\hat{f}_{n}(\omega)\right|^{2} d \omega \geq C_{1}
$$

for all $n$, then

$$
\operatorname{gapr}\left(\left\{f_{n}\right\}_{n=1}^{N}\right) \geq \sqrt{\frac{C_{1} N}{T_{s}}}
$$

for all $N$

Proof: The claim follows from the simple calculation

$$
\begin{aligned}
T_{s}\left(\operatorname{ess}_{\sup } \sup _{t \in[0,1]} \sum_{n=1}^{N}\left|f_{n}(t)\right|^{2}\right) & \geq \int_{0}^{T_{s}} \sum_{n=1}^{N}\left|f_{n}(t)\right|^{2} d t \\
& \geq N C_{1},
\end{aligned}
$$

where in the sum $\sum_{n=1}^{N}\left|f_{n}(t)\right|^{2}$ we replace $f_{n}$ with $\hat{f}_{n}$ if $\hat{f}_{n}$ satisfies inequality (19) and $f_{n}$ does not satisfy (18).

One would then like to determine conditions that result in overlap among signals. Here we have the following: if the functions $\left\{f_{n}\right\}_{n=1}^{\infty}$ are uniformly bounded, then there must exist subsets of $[0,1]$ where large numbers of them have large absolute value, such that the sum $\sum_{n=1}^{N}\left|f_{n}(t)\right|^{2}$ is large. The following proposition, due to Olevskiı̌, gives a lower bound on the measure of the set for which the supremum norm grows like in line (9).

Theorem 2.8 (Part of Thm 1, Ch. 1, Sec. 2 in [5]): Let $\left\{f_{n}\right\}_{n=1}^{\infty}$ be a set of orthonormal functions in $L^{2}([0,1])$, and assume further that $\left\|f_{n}\right\|_{L^{\infty}([0,1])} \leq M$ for all $n$. Then the set

$$
E=\left\{x \in[0,1]: \limsup _{N \rightarrow \infty} \frac{1}{N} \sum_{n=1}^{N}\left|f_{n}(t)\right|^{2}>0\right\}
$$


has Lebesgue measure $\lambda(E) \geq \frac{1}{M^{2}}$. That is, for all $t$ in some set of measure at least $\frac{1}{M^{2}}$, for any $\epsilon>0$ there exists an infinite set of integers $\left\{N_{k}\right\}_{k=1}^{\infty}$, such that

$$
\left(\sum_{n=1}^{N_{k}}\left|f_{n}(t)\right|^{2}\right)^{1 / 2} \geq \sqrt{N_{k}-\epsilon}
$$

Without this theorem, one could speculate that as $N$ increases there exist bounded orthonormal bases and coefficients such that one can reduce the size of the region where high growth occurs to be arbitrarily small. Olevskiı''s result rules out such behavior and gives a lower bound on the size of the set where large peaks occur. Thus, the behavior $\operatorname{gapr}\left(\left\{f_{n}\right\}_{n=1}^{N}\right) \geq$ $\sqrt{N}$ is never restricted to a set of arbitrarily small measure. Rather, growth of the peak value of order $\sqrt{N}$ occurs on a set of Lebesgue measure at least $\frac{1}{M^{2}}$.

\section{Prolate Spheroidal WaVE Functions}

We now address the PAPR behavior of prolate spheroidal wave functions. These functions were first studied intensively in communications engineering by Landau, Pollak and Slepian [6], [3]. They were then used by Wyner to prove Shannon's fundamental theorems of information theory using realizable transmission signals [7]. In order to introduce them we require another definition.

Definition 3.1: A function $f \in L^{2}(\mathbb{R})$ is band-limited to $[-\Omega, \Omega]$ if there exists a function $F \in L^{2}(\mathbb{R})$ such that

$$
f(t)=\frac{1}{2 \pi} \int_{-\Omega}^{\Omega} F(\omega) e^{i \omega t} d t
$$

where equality is in $\|\cdot\|_{L^{2}(\mathbb{R})}$. We denote these functions $\mathcal{B}$.

Theorem 3.2: ([6], [3]) For any $T>0$ and $\Omega>$ 0 , there exists a countably infinite sequence of functions $\psi_{0}(t), \psi_{1}(t), \psi_{2}(t), \ldots$ and a set of real positive numbers $\lambda_{0}>\lambda_{1}>\ldots$ with the following properties:

1) The $\psi_{n}(t)$ are band-limited to $[-\Omega, \Omega]$, orthonormal on the real line and complete in $\mathcal{B}$ :

$$
\int_{-\infty}^{\infty} \psi_{n}(t) \psi_{j}(t) d t=\delta_{i, j} \text { for all } i, j .
$$

2) In the interval $[-T / 2, T / 2]$ the $\psi_{n}(t)$ are orthogonal and complete in $L^{2}([-T / 2, T / 2])$ :

$$
\int_{-T / 2}^{T / 2} \psi_{n}(t) \psi_{j}(t) d t=\lambda_{j} \delta_{i, j} \text { for all } i, j .
$$

3) For all $t$, real or complex,

$$
\lambda_{n} \psi_{n}(t)=\int_{-T / 2}^{T / 2} \frac{\sin \Omega(t-s)}{\pi(t-s)} \psi_{n}(s) d s \text { for all } i \text {. }
$$

These functions are called the prolate spheroidal wave functions (PSWFs).

The prolate spheroidal wave functions have the following localization property: the first $N$ such functions are the $N$ orthonormal functions in $\mathcal{B}$ that maximize the sum

$$
\sum_{n=1}^{N} \int_{-T / 2}^{T / 2}\left|f_{n}(t)\right|^{2} d t
$$

This concentration made them suited for Wyner's work on the capacity of the band-limited Gaussian channel. The theorem below shows that the possible PAPR for transmission schemes using these signals can grow proportional to the square-root of the bandwidth.

Proposition 3.3: Given any $\delta>0$, there exists a constant $C_{\delta}$, such that for any time interval $[-T / 2, T / 2]$ and frequency interval $[-W / 2, W / 2]$ satisfying $2 T W>C_{\delta}$, the prolate spheroidal wave functions corresponding to $T$ and $W$ satisfy

$\operatorname{ess.sup}_{t \in[-T / 2, T / 2]}\left(\sum_{n=1}^{2 T W(1-\delta)}\left|\psi_{n}(t)\right|^{2}\right)^{1 / 2} \geq(1-\delta) \sqrt{2 W}$.

Proof: For $f \in L^{2}(\mathbb{R})$ we define $D_{T} f(t)=f(t)$ for $t \in[-T, T]$ and $D_{T} f(t)=0$ for $t \notin[-T, T] . \mathcal{F}$ denotes the Fourier transformation. We have that for any fixed $\delta>$ 0 , that $\lambda_{2 T W(1-\delta)} \rightarrow 1$ as $T W \rightarrow \infty$ (See Section 4.3 in [7]). So, given $\delta>0$, there exists a constant $C_{\delta}$, such that $\lambda_{2 T W(1-\delta)} \geq 1-\delta$ for all $T W \geq C_{\delta}$. Using properties given in Section 4.3 in [7] we have

$$
\begin{aligned}
\text { ess.sup }_{t \in[-T / 2, T / 2]} \sum_{n=1}^{2 T W(1-\delta)}\left|\psi_{n}(t)\right|^{2} \\
\geq \frac{1}{T} \int_{-T / 2}^{T / 2} \sum_{n=1}^{2 T W(1-\delta)}\left|\psi_{n}(t)\right|^{2} \\
=\frac{1}{T} \sum_{n=1}^{2 T W(1-\delta)}\left\|D_{T} \psi_{n}\right\|_{L^{2}(\mathbb{R})}^{2} \\
=\frac{1}{T} \sum_{n=1}^{2 T W(1-\delta)}\left\|\mathcal{F} D_{T} \psi_{n}\right\|_{L^{2}(\mathbb{R})}^{2} \\
\geq \frac{1}{T} \sum_{n=1}^{2 T W(1-\delta)}\left\|D_{W} \mathcal{F} D_{T} \psi_{n}\right\|_{L^{2}(\mathbb{R})}^{2} \\
=\frac{1}{T} \sum_{n=1}^{2 T W(1-\delta)} \lambda_{n} \\
\geq 2 W(1-\delta)(1-\delta) .
\end{aligned}
$$

Thus peak values can grow for the prolate spheroidal wave functions like the square-root of the bandwidth. This makes sense because the energy of each such function in the frequency domain is concentrated in the same interval. Nonetheless, this leads to high peaks in the time domain. Of course bandwidth is the significant parameter here, since the capacity increases with the bandwidth.

\section{AN EXAMPLE: DOWNLINK IN DS-CDMA}

In this section we show that DS-CDMA signals satisfy the worst case behavior discussed earlier. We give a very simple construction to show how large peaks can occur. First we introduce the signal system: the Walsh system is the orthonormal basis used in DS-CDMA, and it, in turn, is the completion of the Rademacher system. 
Definition 4.1: The Rademacher functions, denoted $r_{0}, r_{1}, \ldots$, are defined on $[0,1]$ by

$$
r_{k}(t)=\operatorname{sign} \sin \left(2 \pi 2^{k} t\right),
$$

where we define sign $0=-1$. The Walsh functions, denoted $w_{0}, w_{1}, \ldots$ are defined using the Rademacher functions by

$$
w_{0}(t)=1
$$

and

$$
w_{2^{k}+m}(t)=r_{k}(t) \cdot w_{m}(t)
$$

for $k=0,1,2, \ldots$ and $m=0, \ldots, 2^{k}-1$.

In DS-CDMA, the base station transmits the signal

$$
x(t)=\sum_{n=1}^{N} b_{n}(t) w_{n}(t) \quad t \in[0,1],
$$

where

$$
\int_{0}^{1}|x(t)| d t=\sum_{n=1}^{N}\left|b_{n}(0)\right|^{2}
$$

and

$$
\mathbb{E} \int_{0}^{1}|x(t)| d t=\sum_{n=1}^{N} \mathbb{E}\left|b_{n}(0)\right|^{2}=\mathrm{P}_{\text {tot }}
$$

We define a specific signal $x^{*}$ by setting $b_{n}^{*}(0)= \pm \sqrt{\frac{\mathrm{P}_{\text {tot }}}{N}}$. We are interested in the peak value $\sup _{t \in[0,1]}|x *(t)|$. However, we note that if $N \leq 2^{m}$, then $w_{n}(t)=1$ for $t \in\left[0,2^{-m}\right]$ and for $n=1, \ldots, N$. So, if we take $b_{n}^{*}(0)=\sqrt{\frac{\mathrm{P}_{\text {tot }}}{N}}$ for $n=1, \ldots, N$, for $t \in\left[0,2^{-m}\right]$

$$
\begin{aligned}
x^{*}(t) & =\sum_{n=1}^{N} b_{n}^{*}(t) w_{n}(t)=\sqrt{\frac{\mathrm{P}_{\mathrm{tot}}}{N}} \sum_{n=1}^{N} 1 \\
& =\sqrt{N \mathrm{P}_{\mathrm{tot}}}
\end{aligned}
$$

for $t \in\left[0,2^{-m}\right]$. Thus

$$
\operatorname{papr}\left(x^{*}\right)=\frac{\left\|x^{*}\right\|_{L^{\infty}([0,1])}}{\left\|x^{*}\right\|_{L^{2}([0,1])}} \geq \frac{\sqrt{N \mathrm{P}_{\mathrm{tot}}}}{\sqrt{\mathrm{P}_{\mathrm{tot}}}}=\sqrt{N} .
$$

Thus, the downlink portion of a DS-CDMA system has the same PAPR behavior as OFDM systems. We note, additionally, that users with high data rate requirements are allocated multiple spreading sequences, and thus also transmit a sum of Walsh functions. That is, if a user is allocated $k$ spreading functions, the user can generate signals with papr $\sqrt{k}$, and so high papr is not exclusive to the downlink.

\section{CONCLUSION}

We have investigated several properties of the peak behavior of single-user signals and orthonormal systems and shown that in each of these the OFDM system offers best possible performance, even though it, of course, remains a problem. Even though they become spread out, the prolate spheroidal wave functions also can produce high peak values. Lastly, we demonstrated how large peaks can occur in DS-CDMA.

\section{REFERENCES}

[1] H. Boche and V. Pohl. Signal Representation and Approximation Fundamental Limits. European Trans. Telecommun. (ETT), 18(5):445456, Aug. 2007.

[2] B. Kashin and L. Tzafriri. Lower estimates for the supremum of some random processes. East J. Approx., 1(1):125-139, 1995.

[3] H. Landau and H. Pollak. Prolate spheroidal wave functions, fourier analysis and uncertainty - II. Bell Syst. Tech. Journal, 40(1):65-84, 1960.

[4] M. Ledoux. The concentration of measure phenomenon, volume 89 of Mathematical Surveys and Monographs. American Mathematical Society, Providence, RI, 2001.

[5] A. M. Olevskiı̌. Fourier series with respect to general orthogonal systems. Springer-Verlag, New York, 1975. Translated from the Russian by B. P. Marshall and H. J. Christoffers, Ergebnisse der Mathematik und ihrer Grenzgebiete, Band 86.

[6] D. Slepian and H. Pollak. Prolate spheroidal wave functions, fourier analysis and uncertainty - I. Bell Syst. Tech. Journal, 40(1):43-64, 1960.

[7] A. Wyner. The capacity of the band-limited gaussian channel. Bell Syst. Tech. Journal, 40(4):359-395, March 1966. 